\title{
Importance of Lymphoscintigraphy to Find Sentinel Ganglion in the Context of Malignant Neoplasm of Breast
}

Sara Gonçalves ${ }^{1}$, Filipa Gonçalves ${ }^{2}$, Carlota Rodrigues ${ }^{2}$ and Rafael Macedo ${ }^{2^{*}}$

${ }^{1}$ Higher School of Health Technology in Lisbon, Lisbon, Portugal

${ }^{2}$ Nuclear Medicine Service of SESARAM, E.P.E, Funchal, Madeira Island, Portugal

*Corresponding author: Rafael Macedo, Nuclear Medicine Service of SESARAM, E.P.E, Funchal, Madeira Island, Portugal, Tel: 00351291705767; E-mail: rafaelmacedo.mednuclear@gmail.com

Received date: May 07, 2018; Accepted date: May 11, 2018; Published date: May 16, 2018

Copyright: (c) 2018 Gonçalves S, et al. This is an open-access article distributed under the terms of the Creative Commons Attribution License, which permits unrestricted use, distribution, and reproduction in any medium, provided the original author and source are credited.

\begin{abstract}
Aim: To assess the importance of the sentinel ganglion (GS) detection technique by lymphoscintigraphy in order to avoid lymphedema of the upper limbs in malignant neoplasms of the breast.

The sentinel ganglion is the first ganglion of the lymphatic chain to drain the lymph from the primary tumor. The lymphoscintigraphy allows to identify this ganglion, to facilitate its removal by the surgeon and to be analyzed by the pathological anatomy through an extemporaneous analysis in order to avoid axillary emptying in case of negative analysis for metastases.

Material and Methods: All lymphoscintigraphy were performed with the BrigthView Philips model camera at the SESARAM, E.P.E.. All of these studies followed the same protocol according to the EANM guidelines. An anamnesis of the patient was carried out, the explanation of the procedure and the positioning of the patient according to the protocol in force. A dynamic study was performed immediately after administration of the radiopharmaceutical and then static, anterior, oblique and lateral images. After processing the acquired images and marking GS on the skin with the aid of the Europrobe 3 probe, clinical reports were performed. For the analysis of the data, we selected female patients with breast neoplasia, who underwent the examination between June 23, 2017 and March 22, 2018, aged between 33 and 88 years.
\end{abstract}

Results: Through the evaluation of the sample of 56 users we can verify that this technique allowed to avoid axillary emptying in 39 patients. Of the 56 patients, 2 had bilateral breast neoplasia, 33 right breast neoplasm and 21 left breast cancer. Of the 2 patients with bilateral malignant neoplasia, one presented both negative ganglia and the other presented negative right GS and positive left GS and consequent axillary emptying. Of the 33 patients with neoplasia of the right breast 8 , they emptied, and of the 21 patients with left breast neoplasia, 9 performed emptying.

Conclusions: According to the data obtained in the SESARAM E.P.E. Nuclear Medicine study, it was verified that lymphoscintigraphy allowed to avoid axillary emptying in 39 patients, which corresponds to $70 \%$ of the sample. It is confirmed that this diagnostic technique is an asset in the preservation of the lymphatic chain.

The availability of this technique in a hospital regimen for the detection of sentinel ganglion in the Regional Health Service is considered important to avoid the limitations associated with axillary emptying and consequent improvement in the quality of life of these patients".

Keywords: Sentinel ganglion; Lymphoscintigraphy; Pathological Anatomy; Nuclear medicine

\section{Aim}

Our study aims to assess the importance of the technique of detection of the sentinel ganglion (SG) by lymphoscintigraphy in order to avoid lymphedema of the upper limbs in malignant neoplasia of the breast.

In Portugal, breast cancer is the most common neoplasm among women and is the second leading cause of cancer death. Annually, about 6,000 new cases are detected and 1,500 women die from this disease. It is a pathology of great impact in society, not only because of its seriousness, but also because it is an organ that symbolizes motherhood and femininity. But, this neoplasia does not only affect women, about $1 \%$ of men, in Portugal, are also diagnosed with this neoplasia [1].

The concept of SG arose with the aim of improving the staging of patients with breast cancer and reducing the morbidity associated with axillary emptying [2].

The SG is the first ganglion of the lymphatic chain to drain the lymph from the primary tumor. Lymphoscintigraphy allows identifying this ganglion, to facilitate its removal by the surgeon and to be analyzed by the pathological anatomy through an extemporaneous analysis in order to avoid axillary emptying in case of negative analysis for metastases. 
Axillary emptying is responsible for most of the morbidity associated with breast surgeries, with seroma, lymphedema, changes in local sensitivity, prolonged hospitalization, chronic pain and difficulty in mobilizing the arm some of the direct effects. Due to these factors, lymphoscintigraphy arose in order to avoid unnecessary axillary emptying in patients with negative axilla [3].

This examination consists of the detection and surgical biopsy of the first lymph node of the tumor lymphatic drainage. Its development requires a multidisciplinary team, involving surgeons, Nuclear Medicine and Pathological Anatomy [3].

After SG identification and resection, if the histopathological examination reveals that it is not compromised by metastases, then it may be assumed that there are few possibilities of metastasis in other axillary nodules located distally and that the tumor is possibly confined to its primary location. However, the presence of metastases in SG is a good indicator of need for axillary emptying and a good marker of more advanced disease [3].

\section{Materials and Methods}

All lymphoscintigraphy were performed with the BrigthView Philips model camera at the SESARAM Nuclear Medicine facilities, E.P.E. All of these studies followed the same protocol according to the EANM guidelines.

The patients were transferred from the hospital to the nuclear medicine service to perform lymphoscintigraphy, with surgery scheduled for the next day. Anamnesis of the users was carried out, the explanation of the procedure and the positioning of the users according to the protocol in force.

The recommended dose for malignant neoplasm of the breast may range from 3-9 $\mathrm{mCi}$ to the recommended dose of Nanocoll ${ }^{\circ}$. A dose of $7 \mathrm{mCi}$ in $0.4 \mathrm{ml}$ divided in 4 injections was given in this service. Surgery occurs approximately 24 hours after lymphoscintigraphy.

Administration of 99mTc-Nanocoll (particles of size 5-100 nm) was performed by 4 peri-areolar subcutaneous injections by the nurse with the aid of a nurse and a nuclear medicine technique, with the patient in the supine position in the chamber range.

Immediately after administration of the radiopharmaceutical, a dynamic study of 20 minutes was performed, and then, at 30 minutes and possibly at 60 minutes, static images in anterior-oblique projection $\left(\mathrm{AO} 45^{\circ}\right)$ and lateral (Figure 1) 5 minutes each, in which the patient was pulling the studied breast in order to deprojet the lymphatic chain. The images were acquired with LEGP collimators, $140 \mathrm{keV}$ power peak, and a $20 \%$ power window in a $64 \times 64$ array [4].

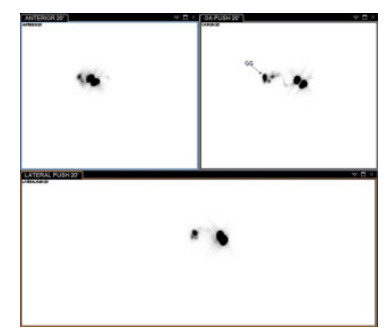

Figure1: Static images acquired 30 minutes after the administration of the radiopharmaceuticals in a patient with right breast neoplasia. The SG is marked by an arrow in the projection AO right side.

In the image processing, the SG is identified in the static image with greater visibility. After its processing the $S G$ is marked with a dermal pen on the skin of the patient by the doctor with the help of the Europrobe 3 probe and the clinical reports are drawn up.

For our study, we selected female patients with breast neoplasia who underwent the examination between June 23, 2017 and March 22, 2018, aged between 33 and 88 years.

\section{Results}

Through the evaluation of the sample of 56 users we can verify that this technique allowed to avoid axillary emptying in 39 patients. Of the 56 patients, 2 had bilateral breast malignant neoplasia, 33 right breast malignant neoplasm and 21 left breast malignant neoplasm (Figure 2). Of the 2 patients with bilateral malignant neoplasia, 1 presented both negative ganglia and the other presented negative SG and negative left SG and consequent axillary emptying. Of the 33 patients with right breast malignant neoplasm, 8 performed emptying, and of the 21 patients with left breast malignant neoplasia, 9 underwent emptying (Figure 3).

\section{Mama afetada}

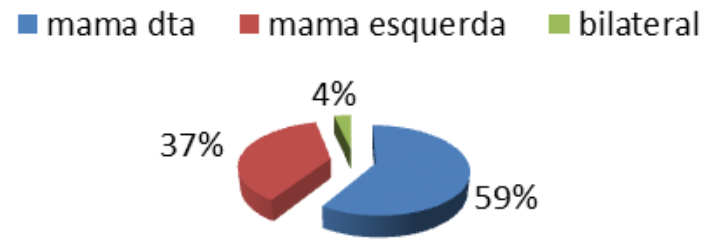

Figure 2: Graph representing the percentages of the laterality of the breast affected by the neoplasia. 


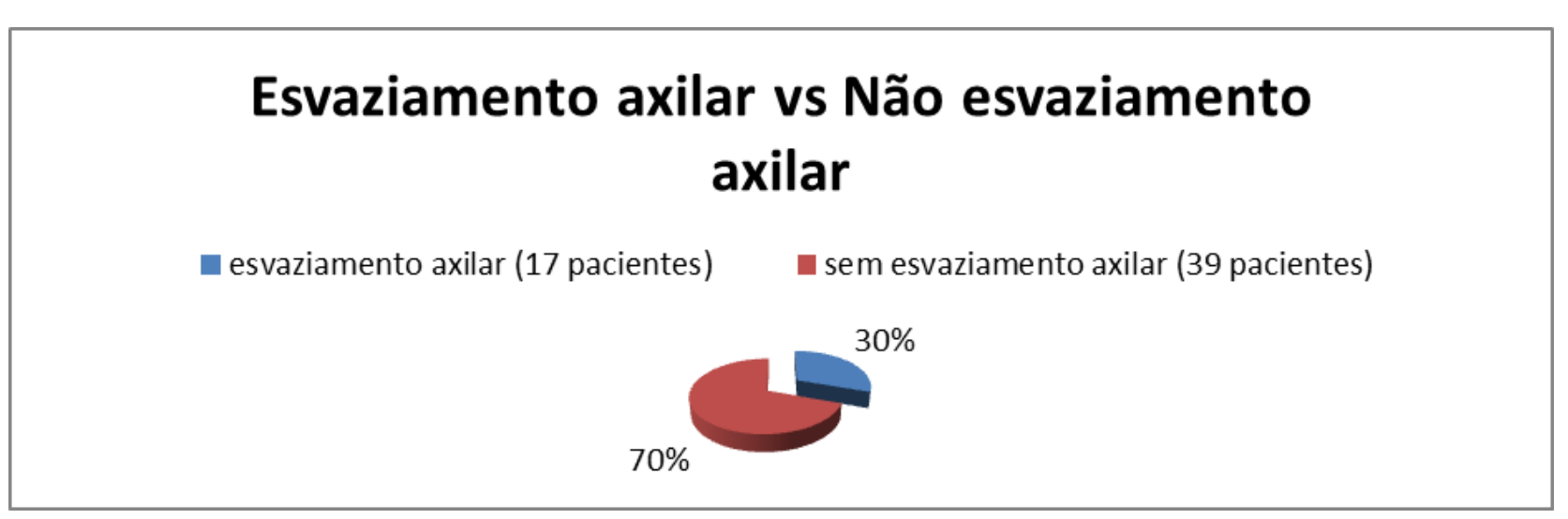

Figure 3: Graph comparing the percentages relative to the number of patients who underwent lymphoscintigraphy and subsequent axillary emptying with respect to the users who did not perform subsequent axillary emptying.

\section{Conclusion}

According to the data obtained in the SESARAM E.P.E. Nuclear Medicine study, it was verified that lymphoscintigraphy allowed to avoid axillary emptying in 39 patients, which corresponds to $70 \%$ of the sample. It is confirmed that this diagnostic technique is an asset in the preservation of the lymphatic chain.

The application of this examination has shown a direct decrease in treatment costs, through the reduction of operative time and hospital stay. Indirectly, it also favorably affects the costs associated with motor and circulatory sequelae that occur after axillary emptying.[3]

The availability of this technique in a public regimen for the detection of SG in the Regional Health Service of Madeira Island is considered important to avoid the limitations associated with axillary emptying and consequent improvement in the quality of life of these patients.

\section{References}

1. https://www.ligacontracancro.pt/

2. Fougo JL, Dinis-Ribeiro M, Dias T, Castro F, Reis P, et al. (2013) The impact of the Sentinel Gang concept on disease-free and global survival and axillary recurrence in patients with Breast Cancer. Rev Port Cardiol. 24: 9-17.

3. Urban CA, Lima RS, Júnior ES, Neto CAH, Yamada A et al. (2000) Sentinel lymph node: A new concept in the surgical treatment of breast cancer. Rev Col Bras Cir. 28: 216-222.

4. Buscombe J, Paganelli G, Burak ZE, Waddington W, Maublant J, et al. (2007) Sentinel node in breast cancer procedural guidelines. Eur J Nucl Med Mol Imaging 34: 2154-2159. 